\title{
L'ALGÉRIE TRANSITION INCERTAINE SUR FOND DE CRISE POLITIQUE ANNONCÉE
}

\author{
Laurence ThieuX \\ Universidad Complutense Madrid
}

\begin{abstract}
Resumen
Tras décadas de inmovilismo político, Argelia conoce desde el 22 de febrero de 2019 profundas transformaciones que cuestionan muchas de las ideas preconcebidas sobre la sociedad argelina. Las manifestaciones masivas, pacíficas y prolongadas de la población argelina contra el sistema político son inéditas y reveladoras de los profundos cambios que en el curso de las dos últimas décadas han transformado tanto la sociedad como el sistema de gobernanza de Argelia. El artículo aporta una reflexión sobre los factores económicos, sociales y políticos que explican la crisis política e institucional y sobre el papel del ejército en este proceso.
\end{abstract}

Palabras clave: Sociedad civil, Ejército Nacional Popular, transición, juventud, redes sociales.

\section{Résumé}

Après des décennies d'immobilisme politique, l'Algérie connaît depuis le 22 février 2019 des bouleversements profonds qui remettent en question bon nombre d'idées reçues sur la société algérienne. Les manifestations massives, pacifiques et prolongées de la population algérienne contre le système politique sont inédites et sont révélatrices des changements profonds qui au cours des dernières décennies ont transformé aussi bien la société que le système de gouvernance de l'Algérie. L'article revient sur les facteurs économiques sociaux et politiques qui expliquent la crise politique et institutionnelle actuelle ainsi que le rôle de l'armée dans ce processus.

Mots clés : Société civile, Armée Nationale Populaire, transition, jeunesse, réseaux sociaux.

Après des décennies d'immobilisme politique, l'Algérie connaît depuis le 22 février 2019 des bouleversements profonds qui remettent en question bon nombre d'idées reçues sur la société algérienne. Les mobilisations populaires, massives, pacifiques, nationales contre le système politique en place ont réussi en quelques mois à faire bouger les lignes d'un « pouvoir » ankylosé dans sa routine autoritaire et complétement déconnecté 
des aspirations, demandes et griefs d'une société algérienne en pleine transformation.

Si au départ le mouvement du 22 février, et ses premières manifestations à Kherrata en Kabylie ${ }^{1}$ visait surtout le cinquième mandat du président Abdelaziz Bouteflika, les demandes des manifestants ont rapidement ciblé toute la classe politique avec l'apparition de slogans dans les manifestations comme «Yetnahaw ga 3 » qui signifie « dégagez tous ».

Après deux décennies à la tête de l'État, le président Bouteflika affaibli par sa maladie depuis 2013 et incapable d'exercer ses fonctions a été finalement lâché par l'armée et forcé à démissionner le 2 avril 20I9, un peu plus d'un mois après le début du soulèvement populaire.

Depuis le début des manifestations, c'est l'armée, colonne vertébrale du pouvoir algérien qui a repris les choses en main et c'est le chef d'État major et vice ministre de la Défense Ahmed Gaïd Salah qui a donné le ton de la réponse $\mathrm{du}$ " pouvoir » en guise de réponse aux citoyens révoltés contre le système politique. Tout en affichant une volonté d'assumer un rôle d'arbitre dans cette confrontation, le chef d'État major, s'exprimant au nom de la " grande muette $»^{2}$ qui n'a jamais été aussi bavarde, a dans le cadre de ses visites aux différentes régions militaires prononcé une série de discours sur la situation politique de l'Algérie.

Cette prolixité de l'armée depuis le début de la crise marque une rupture avec la réserve habituelle de l'armée sur les affaires politiques. À travers ses discours ambigus, l'armée s'est essayée à un exercice d'équilibre compliqué entre d'un côté un soutien manifeste au mouvement populaire tout en prenant ses distances par rapport aux clans du pouvoir accusés de tous les maux et responsables de la crises politique du pays et d'un autre côté comme Institution garante de la stabilité de l'État et ses institutions. En dosant menaces, fermeté, laissez faire et complaisance, l'armée à travers les discours de Gaid Salah, et les éditoriaux de la revue

1 Avant la première manifestation du 22 février qui marque le début du mouvement, les appels à manifester sont apparus à Kherrata en Kabylie le 10 février voir à ce sujet : https://www.lemonde.fr/ afrique/article/2019/06/08/a-kherrata-aux-sources-du-soulevement-algerien_5473351_3212.html

2 Cette expression très utilisée en Algérie pour désigner l'armée, remonte à la IIIe République, en France, période durant laquelle les militaires, étaient privés de droits civiques. 
$\mathrm{E} 1 \mathrm{Djeich}^{3}$ en réponse aux manifestations des étudiants le mardi et du peuple algérien le vendredi ont dit tout et son contraire.

Mais depuis la démission du président le 2 avril, la feuille de route suivie par l'armée est très clairement définie : suivre coûte que coûte la voie tracée par la Constitution : activation de l'article Io2, désignation du Président du Sénat, Bensalah comme président intérimaire pendant 90 jours, maintien du gouvernement Bedoui nommé par le Président Bouteflika au début de la crise après le limogeage de Ouyahia et préparation de nouvelles élections pour le 4 juillet.

L'attachement de l'armée 4 à cette feuille de route et rejetée par l'ensemble du peuple algérien qui en "vendredirant " pacifiquement depuis février a clairement demandé la démission des $3 \mathrm{~B}$, le départ de ceux qui ont contribué à plonger l'Algérie dans la crise politique, et en fin de compte un changement radical de système politique.

Si l'armée avait certainement misé sur l'essoufflement du mouvement avec le début du ramadan elle a du finalement cédé et annuler les élections prévues pour le 4 juillet faute de candidats crédibles et l'échec annoncé d'un scrutin considéré comme illégitime et boycotté dès le départ. Pour l'armée une sortie de crise signifie avant tout le retour à l'ordre mais son obstination à imposer des solutions qui ne répondent en rien aux aspirations démocratiques du peuple algérien ne peut qu'alimenter le mouvement populaire contre le système.

L'armée est aussi restée sourde aux appels au dialogue de certaines personnalités issues du monde politique ou de la société civile ${ }^{5}$.

3 Voir les éditoriaux de la revue E1 Djeich de mars, avril, mai et juin 2019 : https://www.mdn. $\mathrm{dz} /$ site_principal/index.php?L=fr\#undefined

4 Le discours prononcé par Gaïd Salah à Ouargla (4ème région militaire et 4ème visite depuis le début de la crise) illustre l'ambiguité entretenue par rapport au mouvement populaire en mentionnant les risques de manipulation et de dérapage mais réitère aussi l'attachement de l'armée à la feuille de route tracée par la Constitution. Voir à ce sujet https://www.tsa-algerie.com/document-situation-politique-ce-qua-dit-gaid-salah/

5 Le fondateur de la ligue algérienne des droits de l'homme, Ali Yahia Abdelnour, l'ancien ministre Ahmed Taleb-Ibrahimi et le militaire à la retraite Rachid Benyelles ont lancé un appel le 18 mai 2019 au commandement de l'ANP, l'invitant à initier un dialogue " franc et honnête " avec des figures représentatives du mouvement citoyen (Hirak), des partis et des forces politiques et sociales qui le soutiennent. Voir à ce sujet : https://www.elwatan.com/edition/actualite/ali-yahia-abdennour-ahmed-taleb-ibrahimi-et-rachid-benyelles-lance-un-appel-pour-une-solutionconsensuelle-18-05-2019 
En se substituant aux représentants légaux des Institutions de l'État à la légitimité fortement érodée, Ahmed Gaïd Salah s'est aussi situé au cœur de l'impasse politique dans laquelle se trouve l'Algérie quatre mois après le début des manifestations.

\section{COMMENT EN EST ON ARRIVÉ LÀ ?}

Pour déchiffrer la crise politique et institutionnelle de l'Algérie et le rejet massif du peuple algérien du régime politique en place depuis l'indépendance du pays en 1962, il est nécessaire de remonter en arrière et décrire les mécanismes qui ont permis la résilience d'un système de gouvernance durant des décennies et plus particulièrement depuis l'élection en 1999 du président Abdelaziz Bouteflika au lendemain de la décennie noire (Dris Ait Hamadouche, L. y C. DRIS, 20I2).

Au cours des deux dernières décennies, le conglomérat de clans qui se sont partagés le pouvoir et les richesses de l'État en Algérie ont fonctionné en vase clos mais en essayant de maintenir un assez large réseau de clientèles qui cependant. Cet accaparement du pouvoir s'est réalisé à l'ombre d'une architecture institutionnelle de façade où les partis, la justice, le pouvoir législatif et exécutif ont été vidés de leurs pouvoirs au profit de cette gouvernance parallèle dirigée par les réseaux complexes (hommes d'affaires gravitant autour du clan présidentiel, services d'intelligence, hiérarchie militaire) constitués autour des opportunités économiques offertes par l'Etat (contrats publics, ...). Une corruption systémique dilapidant les ressources issues du secteur des hydrocarbures principalement (Serres, 20I9).

Lorigine de cette dérive politico-mafieuse des cercles proches du pouvoir remonte aux années 8o, une décennie de rupture avec une succession de crises qui vont modifier en profondeur les structures du pouvoir en Algérie. Tout d'abord la crise économique provoquée par la chute du prix des hydrocarbures en I986 et qui avait provoqué alors un processus d'endettement inégalé de l'État algérien a obligé la présidence de Chadli Bendjedid à libéraliser le système économique. L'Algérie a connu aussi à la fin des années 80 une crise politique et idéologique profonde, avec une remise en question globale de la légitimité de l'armée au cœur du pouvoir qui réprimait dans le sang les manifestations d'octobre i988. L'ouverture 
politique contrainte par la crise économique et un système politique en perte de légitimité débouchèrent sur l'adoption de la Constitution de 1989 et la fin du parti unique donnant libre cours à l'expression de la diversité identitaire et religieuse de la société algérienne. La montée de l'islam politique en Algérie dans les années 8o et l'arrivée en force du FIS dans le paysage politique algérien à la fin des années 80 précipitèrent la fin de cette courte période de libéralisation politique en Algérie (Thieux, 2008).

Toutes ces crises ont conduit à une remise en questions de la légitimité du pouvoir basé sur une rente historique, économique et sécuritaire (Mañé Estrada, A. Thieux, L. y Hernando de Larramendi, M. 20r6). Le chaos de la guerre civile des années 90 a aussi menée à une reconfiguration politique du pouvoir. Bouteflika à son arrivée à la présidence en I999, parachuté par l'armée pour restaurer une image favorable de l'Algérie à l'extérieur et redonner un pouvoir civil à une Algérie meurtrie par une décennie de violence sauvage produite par l'affrontement entre l'armée et les groupes islamistes armés, ne voulait pas être un 3/4 de président. Depuis son arrivée au pouvoir, il n'a eu de cesse de reconstruire son pouvoir, le consolider en utilisant ses atouts diplomatiques d'un côté et l'opportunité qui se présentait de devenir l'homme de la réconciliation nationale.

Dans ce processus cependant Bouteflika devait composer avec l'armée d'une part et en son sein les services d'intelligence (le DRS) qui au cours de la décennie noire avaient acquis un poids prépondérant. C'est au cours des années 90 que l'armée s'impliqua directement dans la gestion politique du pays après l'interruption du processus électoral en janvier 92 pour éviter une victoire prévisible du FIS au deuxième tour des législatives que l'armée. Son image en est sortie fortement dégradée. Le capital social extérieur de Bouteflika devait permettre à l'Algérie de sortir de l'isolement et revenir sur la scène internationale (Belkaïd, 2009).

La présidence de Bouteflika a cependant été dès le départ entachée par un déficit de légitimité, ses six rivaux à la présidentielle s'étant retirés d'un scrutin en raison du manque de transparence et de liberté. Afin de pallier ce handicap de départ Bouteflika s'emploiera à renforcer sa légitimité vis à vis du peuple et surtout sa position interne vis à vis de l'armée en exploitant ce qui précisément avait décidé l'armée à faire appel à lui. Tout d'abord, son capital social extérieur lui permis de regagner la confiance 
des pays occidentaux, sur la base d'une nouvelle narrative présentant la guerre contre les groupes armés islamistes comme une bataille d'avant garde contre le terrorisme islamiste nouvel ennemi des Etats Unis après les attentats du in septembre. Ensuite il s'agissait de consolider son pouvoir en interne. La Concorde civile en 1999 puis la Charte de réconciliation nationale en 2005 ont renforcé la position du président qui devenait l'homme qui avait réussi à restaurer la paix en Algérie : une narrative qui résiste peu à l'épreuve des faits. On doit en effet les premières initiatives de paix avec les islamistes au Président Zeroual qui en 1997 initiait la Rahma et signait les accords de paix avec l'AIS, la branche armée du FIS. D'autre part loin de constituer un véritable processus de vérité et justice, la charte approuvée par référendum en 2005 ne faisait que tourner la page sur une décennie tout en interdisant de revenir en arrière (Dutour, 2008). Ainsi la Charte criminalise dans son article 46 « quiconque qui, par ses déclarations, écrits ou tout autre acte, utilise ou instrumentalise les blessures de la tragédie nationale, pour porter atteinte aux institutions de la République algérienne démocratique et populaire, fragiliser l'Etat, nuire à l'honorabilité de ses agents qui l'ont dignement servie, ou ternir l'image de l'Algérie sur le plan international $»^{6}$.

Bien qu'essentiels ces deux éléments nétaient pas suffisants pour asseoir le pouvoir du Président et de ses proches au sein d'un système collégial où l'armée avait toujours le dernier mot. La stratégie suivie par Bouteflika a consisté à élargir sa base de pouvoir en diversifiant ses soutiens et en intégrant de nouveaux acteurs économiques émergents après la libéralisation de l'économie algérienne. Ces entrepreneurs qui ont fait des fortunes grâce aux largesses de l'État en matière de contrats publics, avantages fiscaux et autres pratiques plus douteuses et qui sont ciblés aujourd'hui par l'opération main propre de l'armée. Le clan présidentiel a aussi réussi à se défaire des « janviéristes ", les décideurs des années 90. Avec la mise à la retraite du général Lamari qui était à la tête de l'état major de l'armée et en le remplaçant par Gaid Salah, Bouteflika réussis-

6 La Charte pour la Paix et la réconciliation Nationale a été approuvée par 97,36\% des voix et avec une participation de 82,04\%. Ver Thieux, L. https://iecah.org/index.php/articulos/739-la-carta-para-la-reconciliacion-nacional-en-arhttp://www.interieur.gov.dz/index.php/ fr/dossiers/168-la-charte-pour-la-paix-et-la-reconciliation-nationale.html\#faqnoanchorgelia-un-plan-de-amnistia-que-deja-heridas-abiertas 
sait en 2004 son premier coup de force. En 2008 afin de parachever une entreprise de longue haleine le président modifiait la Constitution afin de pouvoir briguer un troisième mandat ce qui sera chose faite en 2009 .

Les « printemps arabes » n’ont pas altéré la continuité politique du régime. L'adoption de réformes superficielles et quelques concessions sans impact n'ont altéré la nature du système politique. L'utilisation du chantage de la peur, le rappel des vieux démons susceptibles de replonger l'Algérie dans un cycle infernal de violence avec en toile de fond les chaos libyen et syrien ont freiné l'émergence d'un front de contestation politique. La manne pétrolière, encore abondante début 201 en raison de la tendance à la hausse du prix des hydrocarbures qui s'est maintenue jusqu'en 20I3, a aussi permis d'acheter la paix sociale à travers une redistribution élargie de la rente qui a calmé pour un temps les revendications économiques et sociales. En Algérie, il faut le rappeler, la population n'a jamais cessé d'exprimer son mécontentement depuis les années 2000, et principalement sous forme de micro-révoltes localisées (Thieux, L. y Hernando de Larramendi, M. 2019).

La permanence de cette insatisfaction sociale qui touche particulièrement les régions périphériques de l'Algérie est révélatrice des déficiences des politiques publiques mises en place, incapables d'enclencher un processus de développement durable sur l'ensemble du territoire national malgré l'abondance des ressources naturelles disponibles et les richesses accumulées par l'État (Belakhdar, 2015). Ces révoltes sont aussi le signe de l'absence de mécanismes de médiation entre ceux qui dirigent le pays et la société.

Après des décennies de répression et autres stratégies insidieuses déployées par le pouvoir la société civile algérienne a été affaiblie et neutralisée. Les organisations qui ont essayé de créer des espaces d'expression et d'action autonomes vis à vis du pouvoir ont été systématiquement privées de ressources et d'espaces pour mener à bien leurs activités (REMDH, 2OI2).

Ces associations, ${ }^{7}$ qui ont eu le mérite de résister dans un contexte très hostiles sont toujours présentes et depuis le 22 février elles ont re-

7 Le groupe solidarité Algérie représente en partie ce noyau d'organisation qui s'est toujours maintenu dans l'opposition au système (Le collectif des familles de disparus, RAJ, LADDH, les Syndicats Autonomes regroupés autour de la Conféderation des syndicats algériens (CSA), le réseau 
joint le mouvement populaire et l'accompagne dans sa structuration. Ces organisations regroupées autour du collectif de la société civile tentent aujourd'hui de devenir des forces de proposition pour la période de transition. Le Collectif de la dynamique de la société civile pour une transition démocratique ; la dynamique autour de la Confédération des syndicats algériens et le collectif civil pour le changement qui essayent actuellement de fédérer et articuler leurs propositions afin de proposer une seule feuille de route pour une sortie pacifique de la crise ${ }^{8}$.

Cette société civile empêchée par les tracasseries administratives et les harcèlements fréquents des forces de sécurité est aujourd'hui en train de récupérer le temps perdu, en se réappropriant l'espace public, en redynamisant le débat et en essayant de jeter des ponts avec une jeunesse qui a été au cœur du mouvement populaire depuis le début.

Cette jeunesse algérienne que l'on découvre dans toute son ingéniosité, sa détermination et son intelligence a réussi à marquer une nouvelle étape de la vie politique algérienne. Les jeunes en Algérie sont nombreux (les moins de 25 ans représentent $40 \%$ de la population), mieux éduqués. Il faut rappeler ici que le nombre d'étudiants en Algérie à été multiplié par 4 en 20 ans : ils étaient en 1999 425.000 et il y a maintenant en Algérie I,7 millions d'étudiants dont un tiers sont des filles 9 . La pénétration rapide d'Internet, des Smartphones et des réseaux sociaux ont aussi permis à cette jeunesse de trouver des espaces d'expression alternatifs sur le net.

\section{CONCLUSIONS}

Le face à face qui oppose l'armée et le mouvement populaire en Algérie qui rejette la feuille de route proposée par le pouvoir parce que celle-ci ne permet pas d'assurer un véritable changement du système au pouvoir. Aucune concession significative, trois mois après le début du soulèvement populaire, n'a été faite par le pouvoir. Les mêmes personnalités sont encore en place : le premier ministre Bédoui, le président intérimaire

des avocats, entre autres. Voir à ce sujet : https://euromedrights.org/fr/membres/

8 Ces trois dynamiques ont tenues plusieurs réunions qui ont réuni 71 associations et syndicats, Voir à ce sujet : https://www.tsa-algerie.com/la-societe-civile-face-a-une-responsabilite-historique/

9 Meyer, J.B (2019): “ Les étudiants, clé du changement en Algérie, Le Monde (11/03), disponible en https:/www.lemonde.fr/afrique/article/2019/03/11/les-etudiants-cle-du-changementen-algerie_5434510_3212.html?xtmc=etudiants_algerie\&xtcr=10 
Bensalah et institutions sont toujours aux commandes et ne peuvent garantir le changement escompté.

Malgré les déclarations favorables au mouvement, les détentions fracassantes des proches du clan Bouteflika et des ex-responsables des services de sécurité ${ }^{10}$ et l'opération main propre contre les oligarques proche du pouvoir ${ }^{11}$, les dénonciations de complots et les règlements de compte ne répondent pas non plus aux aspirations démocratiques du peuple algérien qui demande clairement une transformation profonde du système de gouvernance. Le processus sera certainement long et semé d'embuches. Même si la société civile est affaiblie, elle a montré son dynamisme depuis le début des marches du Hirak et elle est essentielle au succès du processus. Il n'est pas vain de rappeler ici le rôle clef que la société civile tunisienne à jouer dans le processus de transition : un rôle de veille, de proposition et de guide pour atteindre les objectifs de la « révolution ». Mais en Algérie, l'armée est le pilier du système et elle n'entend pas céder dans un contexte régional et international qui s'incline plutôt vers la contre-révolution que vers les démocraties incertaines.

\section{RÉFÉRENCES BIBLIOGRAPHIQUES}

Belakhdar, Naoual. « « L'éveil du Sud » ou quand la contestation vient de la marge. Une analyse du mouvement des chômeurs algériens ", Politique africaine, vol. 137, $\mathrm{n}^{\circ} \mathrm{I}, 2 \mathrm{O} 5$, pp. 27-48.

Belkaï̀, Aкram. « La diplomatie algérienne à la recherche de son âge d'or. » Politique étrangère, 2009, $\mathrm{n}^{\circ}$ 2, pp. 337-344.

Dris Aït Hamadouche, Louisa; Dris, Chérif. « De la résilience des régimes autoritaires: la complexité algérienne ». L'année du Maghreb, 20I2, no VIII, p. 279-30I.

10 Le frère de l'ancien président Abdelaziz Bouteflika, et les ex-patrons du renseignement - Mohamed Mediène, dit Toufik, et Athmane Tartag, dit Bachir ont été arrêtés en mai 2019 pour complot contre l'armée. https://www.jeuneafrique.com/771049/societe/algerie-said-bouteflika-et-deux-ex-patrons-du-renseignement-places-en-detention-provisoire/

$11 \mathrm{https} / / / w w w . l e p o i n t . f r / a f r i q u e / l e s-d e s s o u s-d e-1-o p e r a t i o n-m a i n s-p r o p r e s-e n-a l g e-$ rie-01-05-2019-2310290_3826.php 
Dutour, Nassera. «Algérie: de la Concorde civile à la Charte pour la Paix et la Réconciliation nationale: amnistie, amnésie, impunité ", Mouvements, 2008, $\mathrm{n}^{\circ}$ I, pp. I44-I49.

Mañé Estrada, Aurèlia; Hernando de Larramendi, Miguel; Thieux, Laurence. Argelia en la encrucijada: condicionantes, tendencias y escenarios, Fundación Alternativas, 2016.

REMDH, Rapport Algérie : "Réformes politiques » ou verrouillage supplémentaire de la société et du champ politique en Algérie?, 20I2, disponible en : https://euromedrights.org/fr/publication/rapport-algerie-reformes-politiques-ou-verrouillage-supplementaire-de-la-societe-et-du-champ-politique-en-algerie/

Serres, Thomas. L'Algérie face à la catastrophe suspendue. Gérer la crise et blâmer le peuple sous Bouteflika (1999-20I4). Karthala, 2019.

Thieux, Laurence et Hernando de Larramendi, Miguel. “2. Transformaciones y procesos políticos y sociales en el Norte de África desde 20II”. Informe África, p. 23.

Thieux, Laurence. "Sociedad civil y cambio político y social en Argelia: evolución de discursos y estrategias". Revista de Estudios Internacionales Mediterráneos, 2018. I0.15366/reim2018.25.005

Thieux, Laurence. Islamismo y democracia en Argelia: Francia y Estados Unidos frente al conflicto, Ediciones del Oriente y del Mediterráneo, Madrid, 2008. 